\title{
What hepatologists need to know about COVID-19?
}

\author{
Suna Yapali \\ Department of Gastroenterology, Acibadem Mehmet Ali Aydinlar University Faculty of Medicine, Istanbul, Turkey
}

\section{Introduction}

The world recognized severe acute respiratory syndrome coronavirus 2 (SARS-CoV-2) from Wuhan in December 2019, and soon after, the virus rapidly spread throughout the world and became a global health threat. Coronavirus Disease (COVID-19) is a respiratory disease, which arises from the SARS-CoV-2. COVID-19 may involve so many systems, including respiratory tract, cardiovascular system, gastrointestinal tract and liver. Hepatologists have involved with COVID-19 disease by sustaining the outpatient clinics for elective patients to prevent the spread of SARS-CoV-2; at the same time, maintained the care of patients, such as decompensated cirrhosis and liver transplant patients, in whom the care should not be delayed. In addition, we helped our colleagues in the frontline by consulting the liver involvement of COVID-19. Based on the available scientific data, professional liver societies promptly provided recommendations, which are continuously being updated ${ }^{[1,2]}$

SARS-CoV-2 is a single-stranded RNA virus that replicates using a virally-encoded RNA-dependent RNA polymerase. Angiotensin-converting enzyme 2 (ACE2) is the functional receptor of the SARS-CoV-2 virus, which mediates virus binding and entry to the target cell. ${ }^{[3,4]}$ ACE2 is expressed in vascular endothelium, renal and cardiovascular tissue, and epithelia of the small intestine and testes. ${ }^{[5]}$ ACE2 is also present in biliary and liver epithelial cells; and therefore, may lead to liver dysfunction. ${ }^{[6]}$ Concerning liver dysfunction observed in patients with COVID-19, questions arise whether the pathogenic mechanism is a direct virus-induced cytopathic effect and/or immune damage and/ or hepatotoxicity of the medications. ${ }^{[7,8]}$ Furthermore, the potential risk of severe COVID-19 in patients with chronic viral hepatitis, autoimmune hepatitis, cirrhosis, decompensated cirrhosis, patients with hepatocellular carcinoma (HCC) and liver transplant recipients has been a concern both for the physicians and the patients. Based on the existing data, the involvement of the liver in COVID-19, the risk and severity of COVID-19 in patients with chronic liver disease and the recommendations of professional liver societies will be summarized.

\section{COVID-19 and Liver Involvement}

The studies from China and the United States (U.S.) reported an incidence of serum aminotransferase elevation in hospitalized patients with COVID-19 ranging from $14 \%$ to $53 \% .^{[7,9-14]}$ There is generally mild elevation of aminotransferases, and higher levels are generally

Received: May 11, 2020; Accepted: May 12, 2020; Available online: May 21, 2020 Corresponding author: Suna Yapali; Acibadem Mehmet Ali Aydinlar Universitesi Tip Fakultesi, Gastroenteroloji Bilim Dali, Istanbul, Turkey e-mail: sunayapali@yahoo.com

(c) (i) (s) OPEN ACCESS

cc. This work is licensed under a Creative Commons Attribution-NonCommercial 4.0 International License. (C) Copyright 2020 by Hepatology Forum - Available online at www.hepatologyforum.org associated with severe cases. A retrospective analysis of patients from Wuhan examining the clinical course and risk factors for mortality reported higher mortality in patients with alanine aminotransferase (ALT) elevation, reduced platelets and reduced albumin levels at the time of admission. ${ }^{[15]}$ This is more likely to be a result of reactive immune response characterized by a cytokine storm with multi-organ failure, rather than liver injury. If the pattern of hepatocellular injury is aspartate aminotransferase predominant, this may suggest myositis, cytokine release syndrome, ischemia/hypotension, or a drug-induced liver injury. ${ }^{[12,16,17]}$ Hypoalbuminemia observed in COVID-19 cases is generally related to the severe inflammatory response. ACE2 receptors are also expressed in the cholangiocytes, and elevation of gamma-glutamyl transferase- a biomarker for cholangiocyte injury - was reported in 54\% of hospitalized patients with COVID-19. ${ }^{[13]}$ It is unclear whether a direct virus-induced cytopathic effect and/or immune damage are responsible for the liver function test abnormalities. ${ }^{[7,8]}$ Interestingly, postmortem examination of a patient with acute respiratory distress syndrome did not show viral inclusions in the liver and the histology showed moderate microvesicular steatosis and mild lobular and portal activity, indicating an injury caused by either SARS-CoV-2 infection or drug-induced liver injury. ${ }^{[17]}$

Hepatotoxicity of medications is a key consideration within the differential diagnosis of liver function test abnormalities. There are currently no approved drugs for SARS-CoV-2, and clinical trials are ongoing. Using the experience of China and Europe, each country implemented different treatment protocols. Antiviral efficacy of chloroquine is through the interference with the cellular receptor ACE2 and endosomal acidification of the fusion of the virus. ${ }^{[18-20]}$ Hydroxychloroquine is widely used in the treatment of patients with severe COVID-19 as monotherapy, but also in combination with azithromycin. Hydroxychloroquine and azithromycin have not been associated with ALT abnormalities and an extremely rare cause of acute liver injury. Lopinavir/ ritonavir are approved protease inhibitors (PIs) for human immunodeficiency virus, which promised some hope by showing in vitro antiviral effect on SARS-CoV-2. ${ }^{[1]}$ However, after the results of a clinical trial of patients with severe COVID-19 that showed no proven efficacy of lopinavir/ritonavir compared to no treatment, ${ }^{[22]}$ its use is discontinued in many centers. The risk of lopinavir/ritonavir-associated hepatotoxicity is low. Favipiravir is an RNA polymerase inhibitor, approved for influenza in Japan and Turkey also got the supply of favipiravir from China. Favipiravir is metabolized by aldehyde oxidase and xanthine oxidase. CYP450 iso-enzymes are not involved in metabolism. Transaminases may increase during favipiravir treatment. ${ }^{[19]}$ Remdesivir is a nucleoside analogue/viral RNA polymerase inhibitor that inhibits SARS-CoV-2 in vitro, ${ }^{[23]}$ remdesivir may be hepatotoxic. The clinical trials for the efficacy and safety of remdesivir are ongoing. Tocilizumab -humanised monoclonal antibody, which targets interleukin-6 receptor - is recommended for the treatment of cytokine release syndrome 
in severe cases. ${ }^{[24]}$ Tocilizumab may be hepatotoxic and may increase transaminase levels, but apparent liver injury with jaundice is rare. ${ }^{[25]}$

COVID-19 is also associated with thromboembolic events, which should also be considered in patients with liver function test abnormality. COVID-19 may predispose patients to thromboembolic disease, especially in patients with underlying liver disease. ${ }^{[26]}$

The approach to a patient with COVID-19 who has an abnormality of liver function tests should include;

- Exclusion of other viral etiologies (including hepatitis A, B, C, Cytomegalovirus, Epstein-Barr virus and Herpes Simplex virus).

- Hepatotoxicity of medications should be reviewed. ${ }^{[27]}$

- Abdominal imaging should be considered if there is clinical suspicion for biliary tract obstruction or hepatic/portal venous thrombosis.

In most cases, aminotransferase elevation is transient, but a close follow-up of liver function tests is required. The worsening of the liver function is generally related to immune-mediated inflammation, myositis, ischemia and hypoxia and the cytokine release syndrome, and drug hepatotoxicity. In these cases, the risk of drug hepatotoxicity should be weighed against the benefits of treatment on a case-by-case basis.

\section{Patients with Compensated Liver Disease}

In April 2020, the CDC reported the underlying health conditions among 122,653 U.S. COVID-19 cases. Data for underlying health problems were available in 7,162 (5.8\%) of the patients. Of these, 2,692 $(37.6 \%)$ patients had one or more underlying health conditions or risk factor. Patients with an underlying health problem were more likely to require intensive care unit (ICU) and hospitalization. The most commonly reported conditions were diabetes mellitus, chronic lung disease, and cardiovascular disease. Only 41 patients $(0.6 \%)$ had chronic liver disease, including seven who required ICU admission. ${ }^{[28]}$

There is no evidence for increased risk and severe course of COVID-19 for patients with chronic viral hepatitis. Data from China, a highly endemic country for hepatitis B, showed that chronic hepatitis B does not affect the outcome of COVID-19. ${ }^{[9]}$ The follow-up and refill prescriptions of the patients with chronic hepatitis can be done by telemedicine, and drug adherence should be assessed in patients on antiviral treatment. The initiation of antiviral treatment for chronic hepatitis B and $\mathrm{C}$ is not immediately required for patients with COVID-19, except in patients with hepatitis B flare or patients with hepatitis B who will receive immunosuppressive therapy. ${ }^{[1]}$

Metabolic-associated fatty liver disease (MAFLD) is strongly associated with the features of metabolic comorbidities, such as diabetes, hypertension and obesity, which increases the risk of COVID-19. A small study from China reported that the presence of obesity in MAFLD patients was associated with a $\sim 6$-fold increased risk of severe COVID-19. ${ }^{[29]}$

For patients with autoimmune liver disease, professional liver societies recommend to continue the immunosuppressive treatment without reducing the dose..$^{[1,2]}$ It should be kept in mind, reducing or discontinuing immunosuppressive treatment may result in a flare in patients with autoimmune hepatitis. Dose reductions can be considered, in case of medication-induced lymphopenia, bacterial/fungal superinfections. ${ }^{[2]}$

In patients with compensated cirrhosis, surveillance for HCC and screening for varices are important follow-up measures. During the pandemic, HCC surveillance can be delayed; AASLD considers a 2-month delay reasonable. HCC surveillance should be prioritized for patients with high alpha-fetoprotein levels. At the time of returning to normal outpa- tient visits, the appointments of these patients should be first scheduled. For patients with COVID-19, HCC surveillance should be postponed until after the recovery. The screening for varices should be stratified according to thrombocyte count or Baveno VI criteria. ${ }^{[30]}$ Since endoscopic procedures pose a high risk for spreading of virus-containing droplets, elective endoscopies should be deferred. If the patient has COVID-19, then, the endoscopic procedure should be limited to gastrointestinal bleeding, bacterial cholangitis or other life-threatening conditions.

Patients with autoimmune liver disease, compensated and decompensated cirrhosis should be vaccinated against Streptococcus pneumonia and influenza. ${ }^{[2]}$ Acetaminophen overdosing and the use of nonsteroidal anti-inflammatory drugs should be avoided in patients with cirrhosis and portal hypertension who has COVID-19. ${ }^{[2]}$

\section{Patients with Decompensated Cirrhosis}

Medical care should be provided by telemedicine or phone visits for minimal exposure to the medical staff. Transplantation should be limited to the patients in whom the short-term prognosis is poor. Prophylaxis of spontaneous bacterial peritonitis and hepatic encephalopathy should be closely followed to avoid admissions. Patients with acute decompensation or acute-on-chronic liver failure should undergo SARS-CoV-2 testing. For patients with acute decompensation with ascites, hepatic encephalopathy and spontaneous bacterial peritonitis, treatment should be maintained, and if needed, admissions should not be delayed. In the case of COVID-19, lopinavir/ritonavir is contraindicated for patients with decompensated cirrhosis based on the HCV experience with PIs. ${ }^{[21]}$

In patients who are actively listed for transplantation, donors and recipients should be tested for SARS-CoV-2. The patients should be informed that a negative swab does not completely rule out infection. Although screening with chest computed tomography (CT) is not routinely recommended, chest $\mathrm{CT}$ can be considered during the pre-transplant workup depending upon the resources of transplant centers. The consent form should include the potential risk for nosocomial COVID-19.

\section{Patients after Liver Transplantation}

It is not clear whether patients after liver transplantation who are on immunosuppressive therapy are under the risk of severe COVID-19. A reactive innate immune response may be responsible for severe clinical manifestations. On the other hand, a high rate of metabolic comorbidities in the post-transplant period may lead to an increased risk of severe COVID-19. A preliminary report of 111 patients from an Italian transplant center reported a 3\% mortality in liver transplant recipients during a 3 -week follow-up period. ${ }^{[31]}$ However, this is a small study with a short-term follow-up, comprising biases of not testing the asymptomatic, mildly symptomatic patients and patients who are not tested. The risk for post-transplant patients will be better clarified with future studies.

The care of stable liver transplant patients can be through telemedicine or phone visits without delay in routine laboratory and drug level testing. The immunosuppressive treatments should not be decreased unless there is medication-induced lymphopenia or bacterial/fungal superinfection in case of severe COVID-19. Liver biopsy should be considered if there is a suspicion for acute rejection.

For treatment considerations, lopinavir/ritonavir should not be co-administered with m-TOR inhibitors (sirolimus, everolimus) and drug 
level of calcineurin-inhibitors (cyclosporin, tacrolimus) should be monitored closely. ${ }^{[27]}$

\section{Patients with Hepatocellular Carcinoma}

Systemic treatments and evaluation for liver transplantation should be considered by minimal exposure to medical staff. In patients with compensated cirrhosis and COVID-19, European Association for the Study of Liver (EASL) recommends to postpone locoregional therapies and temporarily withdraw immune-checkpoint inhibitor therapy. ${ }^{[2]}$ For the dosing and continuation of kinase inhibitors in non-severe COVID-19 cases, treatment can be individualized in tumor board meetings. ${ }^{[2]}$

\section{Conclusion}

During the COVID-19 pandemic, at first, the healthcare workers had the concerns for the global shortage of personal protective equipment (PPE) to ensure the continuity of care. In the following months, we realized that it is uncertain how long this pandemic will last. As long as the pandemic is started to be under control in some countries, efforts started to return to normal. Our first goal is to prevent our patients and medical staff from COVID-19 by the precautions of hygiene, social distancing in the outpatient clinics and wards, optimal use of PPE and then maintain care for patients with liver disease to prevent complications that will arise from delaying patient care. With the growing body of future evidence, hepatologists will be more confident in the management of the patients during the pandemic.

\section{References}

1. Fix OK, Hameed B, Fontana RJ, Kwok RM, McGuire BM, Mulligan DC, et al. Clinical Best Practice Advice for Hepatology and Liver Transplant Providers During the COVID-19 Pandemic: AASLD Expert Panel Consensus Statement. Hepatology 2020. [Epub ahead of print]

2. Boettler T, Newsome PN, Mondelli MU, Maticic M, Cordero E, Cornberg $\mathrm{M}$, et al. Care of patients with liver disease during the COVID-19 pandemic: EASL-ESCMID position paper. JHEP Rep 2020;2(3):100113.

3. Lan J, Ge J, Yu J, Shan S, Zhou H, Fan S, et al. Structure of the SARSCoV-2 spike receptor-binding domain bound to the ACE2 receptor. Nature. 2020. [Epub ahead of print]

4. Li W, Moore MJ, Vasilieva N, Sui J, Wong SK, Berne MA, et al. Angiotensin-converting enzyme 2 is a functional receptor for the SARS coronavirus. Nature 2003;426(6965):450-454.

5. Hamming I, Timens W, Bulthuis ML, Lely AT, Navis G, van Goor H. Tissue distribution of ACE2 protein, the functional receptor for SARS coronavirus. A first step in understanding SARS pathogenesis. J Pathol 2004;203(2):631-637.

6. Chai X, Hu L, Zhang Y, Han W, Lu Z, Ke A, et al. Specific ACE2 expression in cholangiocytes may cause liver damage after 2019-nCoV infection. BioRxiv 2020. [Epub ahead of print]

7. Fan Z, Chen L, Li J, Tian C, Zhang Y, Huang S, et al. Clinical features of COVID-19 related liver damage. MedRxiv 2020. [Epub ahead of print]

8. Gu J, Han B, Wang J. COVID-19: Gastrointestinal manifestations and potential fecal-oral transmission. Gastroenterology 2020. [Epub ahead of print]

9. Guan W-J, Ni Z-Y, Hu Y, Liang W-H, Ou C-Q, He J-X, et al. Clinical characteristics of Coronavirus Disease 2019 in China. N Engl J Med 2020. [Epub ahead of print]

10. Chen N, Zhou M, Dong X, Qu J, Gong F, Han Y, et al. Epidemiological and clinical characteristics of 99 cases of 2019 novel coronavirus pneumonia in Wuhan, China: a descriptive study. Lancet 2020;395(10223):507-513.

11. Huang C, Wang Y, Li X, Ren L, Zhao J, Hu Y, et al. Clinical features of patients infected with 2019 novel coronavirus in Wuhan, China. Lancet 2020;395(10223):497-506.
12. Xu L, Liu J, Lu M, Yang D, Zheng X. Liver injury during highly pathogenic human coronavirus infections. Liver Int 2020. [Epub ahead of print]

13. Zhang C, Shi L, Wang FS. Liver injury in COVID-19: Management and challenges. Lancet Gastroenterol Hepatol 2020;5(5):428-430.

14. Richardson S, Hirsch JS, Narasimhan M, Crawford JM, McGinn T, Davidson KW, et al. Presenting Characteristics, Comorbidities, and Outcomes Among 5700 Patients Hospitalized With COVID-19 in the New York City Area. JAMA 2020:e206775.

15. Zhou F, Yu T, Du R, Fan G, Liu Y, Liu Z, et al. Clinical course and risk factors for mortality of adult inpatients with COVID-19 in Wuhan, China: a retrospective cohort study. Lancet 2020;395(10229):1054-1062.

16. Cheung KS, Hung IF, Chan PP, Lung KC, Tso E, Liu R, et al. Gastrointestinal Manifestations of SARS-CoV-2 Infection and Virus Load in Fecal Samples from the Hong Kong Cohort and Systematic Review and Meta-analysis. Gastroenterology 2020:S0016-5085(20)30448-0. [Epub ahead of print]

17. Xu Z, Shi L, Wang Y, Zhang J, Huang L, Zhang C, et al. Pathological findings of COVID-19 associated with acute respiratory distress syndrome. Lancet Respir Med 2020;8(4):420-422.

18. Li G, De Clercq E. Therapeutic options for the 2019 novel coronavirus (2019-nCoV). Nat Rev Drug Discov 2020;19:149-150.

19. Dong L, Hu S, Gao J. Discovering drugs to treat coronavirus disease 2019 (COVID-19). Drug Discov Ther 2020;14(1):58-60.

20. Gautret P, Lagier JC, Parola P, Hoang VT, Meddeb L, Mailhe M, et al. Hydroxychloroquine and azithromycin as a treatment of COVID-19: results of an open-label non-randomized clinical trial. Int J Antimicrob Agents 2020:105949.

21. Casado JL, Del Palacio M, Moya J, Rodriguez JM, Moreno A, Perez-Elías MJ, et al. Safety and pharmacokinetics of lopinavir in HIV/HCV coinfected patients with advanced liver disease. HIV Clin Trials 2011;12(5):235-243.

22. Cao B, Wang Y, Wen D, Liu W, Wang J, Fan G, et al. A Trial of LopinavirRitonavir in Adults Hospitalized with Severe Covid-19. N Engl J Med 2020;382(19):1787-1799.

23. Wang M, Cao R, Zhang L, Yang X, Liu J, Xu M, et al. Remdesivir and chloroquine effectively inhibit the recently emerged novel coronavirus (2019-nCoV) in vitro. Cell Res 2020;30(3):269-271.

24. Mehta P, McAuley DF, Brown M, Sanchez E, Tattersall RS, Manson JJ; HLH Across Speciality Collaboration, UK. COVID-19: consider cytokine storm syndromes and immunosuppression. Lancet 2020;395(10229):1033-1034.

25. Genovese MC, Kremer JM, van Vollenhoven RF, Alten R, Scali JJ, Kelman A, et al. Transaminase levels and hepatic events during tocilizumab treatment: pooled analysis of long-term clinical trial safety data in rheumatoid arthritis. Arthritis Rheumatol 2017;69:1751-1761.

26. Bikdeli B, Madhavan MV, Jimenez D, Chuich T, Dreyfus I, Driggin E, et al. COVID-19 and Thrombotic or Thromboembolic Disease: Implications for Prevention, Antithrombotic Therapy, and Follow-up. J Am Coll Cardiol 2020:S0735-1097(20)35008-7.

27. COVID-19 Drug interactions. Available at: http://www.covid19- druginteractions.org/. Accessed May 5, 2020.

28. CDC COVID-19 Response Team. Preliminary Estimates of the Prevalence of Selected Underlying Health Conditions Among Patients with Coronavirus Disease 2019 - United States, February 12-March 28, 2020. MMWR Morb Mortal Wkly Rep 2020;69(13):382-386.

29. Zheng KI, Gao F, Wang XB, Sun QF, Pan KH, Wang TY, et al. Obesity as a risk factor for greater severity of COVID-19 in patients with metabolic associated fatty liver disease. Metabolism 2020;108:154244.

30. Maurice JB, Brodkin E, Arnold F, Navaratnam A, Paine H, Khawar S, et al. Validation of the Baveno VI criteria to identify low risk cirrhotic patients not requiring endoscopic surveillance for varices. J Hepatol 2016;65(5):899905.

31. Bhoori S, Rossi RE, Citterio D, Mazzaferro V. COVID-19 in long-term liver transplant patients: preliminary experience from an Italian transplant centre in Lombardy. Lancet Gastroenterol Hepatol 2020. [Epub ahead of print] 